\title{
U-shaped inductively coupled feed RFID tag antenna for gain enhancement
}

\begin{abstract}
An inductively coupled feed novel technique is proposed to enhance the performance of RFID tag antennas. The applied feeding concept comprises of two opposing symmetrical Ushaped structures to feed a radiating body. It is a simple alternative to effectively match between the antenna impedance with the chip impedance from the flexible augmentation of equivalent inductance to the radiating body. The proposed feeding method offers better expectation of performance among antenna size, impedance, and gain at the size of $50 \times 70 \times$ $1.6 \mathrm{~mm} 3$. The peak gain of the proposed tag antenna is $2.5 \mathrm{dBi}$ at its operating frequency, which is higher among conventional tag antennas. Measured results indicate that this tag antenna has a good impedance matching characteristic at 904-937 MHz (power reflection coefficient better than $-3 \mathrm{~dB}$ ). The comparison between the simulation and measurement results verified the proposed technique capability to improve overall performance of the tag antennas.
\end{abstract}

Keyword: RFID; Antenna gain; Impedance matching; Inductively coupled feed; Tag antenna 